International Journal of Poultry Science 9 (4): 386-389, 2010

ISSN 1682-8356

(C) Asian Network for Scientific Information, 2010

\title{
Comparison of the Management Characteristics of Sero-positive and Negative Chickens Against Avian Pneumovirus in Uruguay
}

\author{
G. Trenchi' ${ }^{1,2}$, K. Suzuki', M. Petruccelli', G. Giossa ${ }^{1,2}$, G. Rodríguez ${ }^{1,2}$ and H. Trenchi ${ }^{2}$ \\ 'Laboratorio de Diagnóstico de Enfermedades de las Aves y los Pilíferos, \\ Facultad de Ciencias Veterinarias, Universidad Nacional de La Plata, Argentina \\ ${ }^{2}$ Área de Patología y Producción Avícola, Facultad de Veterinaria, \\ Universidad de la República, Montevideo, Uruguay
}

\begin{abstract}
The objective of this study was to investigate the risk factors, especially for management characteristics associated with the seropositivity of avian pneumovirus in broiler chickens Uruguay. Seventeen farms of broiler chickens ( 35 days of age) were studied from October 2008 to April 2009 , comprised data collection through questionnaire interviews for each study farm, in combination with blood sample collections for each chicken $(n=1861)$. Sera were analyzed using a commercial enzyme-linked immunosorbent assay. Univariate and multivariate analyses were used to describe the differences between the two chicken groups categorized according to seropositivity. Percentages of test positive were highly variable between the study farms $(0-81 \%)$ on the basis of different sample sizes (30-224). The final multivariate logistic regression model indicated that five different study farms were less likely to have seropositive-chickens against avian pneumovirus compared with the reference farm ( $81 \%$ of test positive). Chickens introduced from the two unique hatcheries were less likely to be seropositive in comparison with chickens with no hatchery information.
\end{abstract}

Key words: Logistic regression analysis, poultry farming operations, South America

\section{INTRODUCTION}

Avian pneumoviruses have been involved in the upper respiratory tract disease of chickens known as avian rhinotracheitis. The virus has also been associated with swollen head syndrome of chickens. Due to difficulties in isolating and identifying pneumoviruses, serological assays have been developed to confirm infection in commercial chickens. A number of commercial Enzymelinked Immunosorbent Assay (ELISA) kits are available and are used most commonly. Good management practices can notably decrease the severity of avian pneumovirus infection, in particular, optimal ventilation, stocking densities, temperature control, litter quality and biosecurity all have a positive impact on the infection (Cook et al., 1988; Cook, 2000; Cook and Cavanagh, 2002; Gough, 2005). In Uruguay, field investigations to determine seroprevalence of avian pneumovirus were implemented which were simply descriptive studies (Giossa et al., 2010). No quantitative epidemiological investigations to identify risk factors for the infection have been publicized to the best of the authors' knowledge. The objective of this study was to investigate the risk factors, especially for management characteristics associated with the seropositivity of avian pneumovirus in broiler chickens Uruguay.

\section{MATERIALS AND METHODS}

Study area: Uruguay is located in the south-eastern part of South America bordering the South Atlantic Ocean, between Argentina in the west and Brazil in the northeast, with a human population of 3.5 million. Uruguay has a poultry population of 14 million, a poultry meat production of 45,000 tonnes per year and a poultry egg production of 43,600 tonnes per year (FAO, 2009). The south of the country including the capital city Montevideo and Canelones Department has the concentration of chicken population (about $90 \%$ of the total), because of the largest national market Montevideo (Ministerio de Ganadería Agricultura y Pesca, 2009).

Sample collection: Seventeen farms of broiler chickens (35 days of age) were investigated. Each study farm was recruited from the capital city Montevideo, Canelones and Lavalleja (east of Canelones) Departments. None of the chickens had been immunised against avian pneumovirus prior to sampling. The required sample size of 1537 in total from a chicken population of 14 million was sufficient to obtain a $95 \%$ confidence interval (95\% Cl) with a desired precision of $\pm 2.5 \%$ when the estimated seroprevalence was $50 \%$ (Hintze, 2008). The sample size in each of the farms was proportionally

Corresponding Author: K. Suzuki, PROVETSUR, Facultad de Ciencias Veterinarias, Universidad Nacional de La Plata, CC296, La Plata, B1900AWW, Argentina 
assigned (1\% each of the total number of chickens at study farms) by available financial, human and material means. The field study was carried out from October 2008 to April 2009, consisted of data collection through questionnaire interviews for each study farm, in combination with blood sample collections for each chicken. A questionnaire was designed to obtain basic information about management characteristics of the study farms. Major questions related to numbers of broilers (at the visit/shipped to market per year), vaccination programmes conducted, various sanitary measures as well as hatchery of origin.

Laboratory examinations: Blood samples were used for diagnostic tests. Individual-chicken sera were analyzed using a commercial Enzyme-linked Immunosorbent Assay (ELISA) for the detection of antibody against avian pneumovirus (FlockChek@ Avian Pneumovirus Antibody Test Kit, Dr Bommeli AG, a subsidiary of IDEXX Laboratories, Liebefeld-Bern, Switzerland). Positive and negative controls were included for each assay. Absorbance was read on an ELISA reader at $650 \mathrm{~nm}$. Based on the instruction manual of the ELISA kits, serum samples with Sample to Positive $(S / P)$ ratios greater than 0.2 (titres larger than 396) were considered seropositive. An individual serum sample was classified as test positive if the sample had S/P ratio larger than 0.2 .

Data analysis: Data were entered into a database using the Base in the OpenOffice.org software version 3.1.1 (Sun Microsystems, Santa Clara, CA, USA). The statistical analyses were performed using Stata SE 10.1 (Stata Corporation, College Station, TX, USA). Univariate and multivariate analyses were used to describe the differences between the two chicken groups categorized according to seropositivity against avian pneumovirus. Univariate analyses were conducted using Pearson's chi-squared statistic for categorical predictors such as "Farm", "Vaccination programmes" and "Hatchery". Following the univariate analyses, a multivariate logistic regression analysis was conducted to better understand the relationships between the outcome "Seropositivity" and the predictors mentioned above. In the analysis, the most important predictors differentiating between the two chicken groups were identified, based on inclusion of all variables, which were significant at $p<0.10$ in the univariate analysis. A disadvantage of the univariate analysis was that a set of variables, of which each is weakly associated with the outcome, can become important predictors when they are taken together. To prevent this, a significance level that was relatively safe $(p<0.10)$ was selected (Noordhuizen et al., 2001). A stepwise backward variable selection approach was used based on the likelihood-ratio statistic and entry and removal probabilities of $p<0.05$ and 0.10 , respectively. The model goodness-of-fit was assessed using the Hosmer-Lemeshow goodness-of-fit test. The test $p$-value closer to 1 indicates better fit. All variables included in the final regression model were screened for possible interactions and in case of continuous variables for non-linearity of effects (Katz, 1999).

\section{RESULTS}

The 17 study farms had 187,400 broilers in total at the visit (equivalent to $1 \%$ of the total chicken population in Uruguay). Blood samples collected from 1861 chickens in the study farms were serologically investigated. The statistical precision was improved from $\pm 2.5 \%$ to $\pm 1.3 \%$ because of the eventual total number of samples of 1861 (larger than planned) and the overall percentage of test positive of $9 \%(95 \% \mathrm{Cl}: 8-11 \%$, smaller than expected). Percentages of test positive were highly variable between the study farms $(0-81 \%)$ on the basis of different sample sizes (30-224). Table 1 shows descriptive statistics for percentage of test positive against avian pneumovirus in the study broilers between farms. There were statistical differences for percentage of test positive between farms $(p<0.001)$. Table 2 describes the relationship between seropositivity against avian pneumovirus in the study broilers, and use of vaccination programmes as well as difference of hatcheries where the broilers were introduced. Note that the vaccination programmes are against Marek's disease, infectious bronchitis and infectious bursal disease, not against the four infectious agents mentioned above. The seropositivity against avian pneumovirus in relation to the use of vaccination programmes did not indicate statistical significance ( $p$ $=0.56$ ). While the seropositivity in relation to the difference of hatcheries indicated statistical difference $(p<0.001)$. On the basis of the results of this field investigation (Table 1 in conjunction with Table 2), predictors "Farm" and "Hatchery" were included in the final multiple logistic regression model (Table 3 ). In the process of the analysis, four farms (Farm 2, 5, 12 and 13) were excluded from the model because all the samples were negative (Table 1). The other two farms (Farm 7 and 8 ) were also dropped because of collinearity. Therefore the number of samples fitted to the model was 1383, not 1861 in total. The goodness of fit information is presented in the footnote of Table 3 . The model represented a very good fit to the data $(p=$ 1.00). There were no interactions among the predictors. The final model indicated that Farm 4, 6, 9, 15 and 16 were less likely to have seropositive-chickens against avian pneumovirus compared with Farm 1. Also Farm 17 was four times as likely to have seropositive-chickens as Farm 1. Chickens introduced from Hatchery $A$ and $B$ were less likely to be seropositive in comparison with those with no hatchery information. 
Int. J. Poult. Sci., 9 (4): 386-389, 2010

Table 1: Percentage of test positive against avian pneumovius in broilers in the 17 study farms in Uruguay $(n=1861)$

\begin{tabular}{|c|c|c|c|c|}
\hline \multirow[b]{2}{*}{ Farm } & \multirow[b]{2}{*}{$n$} & \multirow[b]{2}{*}{$\%$ of test positive } & \multicolumn{2}{|c|}{$95 \%$ confidence interval } \\
\hline & & & Lower & Upper \\
\hline 1 & 91 & 81 & 73 & 89 \\
\hline 2 & 89 & 0 & 0 & 0 \\
\hline 3 & 79 & 3 & 0 & 6 \\
\hline 4 & 97 & 3 & 0 & 7 \\
\hline 5 & 100 & 0 & 0 & 0 \\
\hline 6 & 100 & 27 & 18 & 36 \\
\hline 7 & 100 & 4 & 1 & 8 \\
\hline 8 & 113 & 4 & 0 & 7 \\
\hline 9 & 111 & 7 & 2 & 12 \\
\hline 10 & 119 & 6 & 2 & 10 \\
\hline 11 & 200 & 5 & 2 & 7 \\
\hline 12 & 65 & 0 & 0 & 0 \\
\hline 13 & 224 & 0 & 0 & 0 \\
\hline 14 & 223 & 0 & 0 & 1 \\
\hline 15 & 30 & 10 & 0 & 21 \\
\hline 16 & 80 & 31 & 21 & 42 \\
\hline 17 & 40 & 15 & 3 & 27 \\
\hline
\end{tabular}

$\mathrm{Chi}^{2}=738.1,16 \mathrm{df}, \mathrm{p}<0.001$

Table 2: Percentage of test positive against avian pneumovirus in the study broilers classified by selected management characteristics $(n=1861)$

\begin{tabular}{|c|c|c|c|c|}
\hline & \multirow[b]{2}{*}{$\mathbf{n}$} & \multirow[b]{2}{*}{$\%$ of test positive } & \multicolumn{2}{|c|}{$95 \%$ confidence interval } \\
\hline & & & Lower & Upper \\
\hline \multicolumn{5}{|c|}{ Vaccination programmes* } \\
\hline+ & 672 & 10 & 8 & 12 \\
\hline n/a & 1189 & 9 & 7 & 11 \\
\hline \multicolumn{5}{|c|}{$\begin{array}{l}\left(\mathrm{Chi}^{2}=0.34,1 \mathrm{df}, \mathrm{p}=0.56\right) \\
\text { Hatchery }^{\dagger}\end{array}$} \\
\hline A & 958 & 2 & 1 & 3 \\
\hline B & 140 & 7 & 3 & 11 \\
\hline $\begin{array}{l}n / a \\
\left(\mathrm{Chi}^{2}=128\right.\end{array}$ & 763 & 18 & 16 & 21 \\
\hline \multicolumn{5}{|c|}{$\begin{array}{l}\left(\mathrm{Chi}{ }^{2}=128.9,2 \mathrm{df}, \mathrm{p}<0.001\right) \\
+; \text { practicing the same vaccination programmes against Marek's disease, infectious bronchitis and infectious bursal disease (not against } \\
\text { avian pneumovirus), } † \mathrm{~A} \text { and } \mathrm{B} \text {; indicating two different hatcheries } \mathrm{A} \text { and } \mathrm{B}, \mathrm{n} / \mathrm{a} \text {; answers not available }\end{array}$} \\
\hline \multirow[t]{3}{*}{ Table 3: } & $\begin{array}{l}\text { regression mod } \\
\text { re the broiler wa }\end{array}$ & $\begin{array}{l}\text { ng the probability } \\
\text { id hatcheries where }\end{array}$ & $\begin{array}{l}\text { seropositiv } \\
\text { 3) }\end{array}$ & \\
\hline & \multirow[b]{2}{*}{ Odds ratio } & & $95 \%$ confidence interval & \multirow[b]{2}{*}{$\mathrm{P}$} \\
\hline & & Lower & Upper & \\
\hline \multicolumn{5}{|c|}{ Farm 1 (reference) } \\
\hline Farm 4 & 0.01 & 0.002 & 0.03 & $<0.001$ \\
\hline Farm 6 & 0.08 & 0.04 & 0.2 & $<0.001$ \\
\hline Farm 9 & 0.02 & 0.01 & 0.04 & $<0.001$ \\
\hline Farm 15 & 0.03 & 0.01 & 0.09 & $<0.001$ \\
\hline Farm 16 & 0.10 & 0.05 & 0.2 & $<0.001$ \\
\hline Farm 17 & 4.2 & 1.1 & 15.9 & 0.03 \\
\hline \multicolumn{5}{|c|}{ Hatchery (n/a; reference) } \\
\hline Hatchery A & 0.01 & 0.002 & 0.03 & $<0.001$ \\
\hline Hatchery B & 0.01 & 0.003 & 0.03 & $<0.001$ \\
\hline
\end{tabular}

Hosmer-Lemeshow goodness-of-fit statistic $0.0,8 \mathrm{df}, p=1.00$

\section{DISCUSSION}

Detection of avian pneumovirus infections can be limited by the sample size used during flock surveys. If the survey programme includes fewer than 30 samples per population, the risk of missing the detection of the first infected chicken (first index case) is increased. Early detection of a recent infection is dependent on the frequency of sampling, sample size and the test sensitivity. To commence a periodical monitoring programme in a unknown situation, the necessary initial step should be to determine the prevalence of the avian pneumovirus in each farm (IDEXX, 2006). Even the smallest sample size of 30 (the $15^{\text {th }}$ farm) in this study met the expectations above. Therefore, all the 
percentage of test positive in this study can be used to know the situation in each farm. The questionnaire designed was to obtain basic information about poultry farming operations of the study farms, such as use of vaccination programmes, difference of hatcheries, as well as the other eight characteristics (data not shown). The former two variables only as well as the variable "Farm" were assigned to describe the differences between sero- positive and negative chicken groups against avian pneumovirus. The vaccination programmes are against Marek's disease, infectious bronchitis and infectious bursal disease, not against avian pneumovirus. There is a statistical difference between chickens being practiced particular vaccination programmes and chickens not being practiced them, in relation to the seropositivity against avian pneumovirus. Although the vaccination programmes do not include to confer immunity against avian pneumovirus, the chickens with the programmes would be more likely to be practiced the same or similar sanitary measures, which could contribute to prevent the chickens from various infections. No information of 763 chickens regarding the use of any particular hatcheries was obtained. It does not mean that those chickens were not introduced from the hatcheries A or B. Final logistic regression model represented that chickens reared at Farm 17 had significant odds ratio of 4.2 having seropositivity against avian pneumovirus. It seems curious because the percentage of test positive at Farm $17(15 \%)$ was lower than that at reference Farm 1 (81\%). Each study chicken at Farm 17 has hatchery information (odds ratio of 0.01 for Hatchery $B$ ). Therefore the actual odds ratio for the chickens was $0.042(=4.2 * 0.01)$ (Bland, 2000). It proves that each chicken at farm 17 is less likely to have seropositivity against avian pneumovirus.

\section{ACKNOWLEDGEMENTS}

This study was carried out as part of the project for the capacity development for improvement of livestock hygiene in the southern part of South America through regional cooperation [commonly known as: Proyecto de desarrollo professional continuo para los veterinarios del Sur (PROVETSUR)], funded by the Japan International Cooperation Agency.

\section{REFERENCES}

Bland, M., 2000. An Introduction to Medical Statistics. Oxford University Press, Oxford.

Cook, J.K.A., 2000. Avian pneumovirus infections of turkeys and chickens. Vet. J., 160: 118-125.

Cook, J.K.A. and D. Cavanagh, 2002. Detection and differentiation of avian pneumoviruses (metapneumoviruses). Avian Pathol., 31: 117-132.

Cook, J.K.A., C.A. Dolby, D. J. Southee and A.P.A. Mockett, 1988. Demonstration of antibodies to Turkey rhinotracheitis virus in serum from commercially reared flocks of chickens. Avian Pathol., 17: 403410.

FAO, 2009. FAOSTAT. Rome. Available from: http:// faostat.fao.org/.

Giossa, G., K. Suzuki, M. Petruccelli, G. Rodriguez, G. Trenchi and H. Trenchi, 2010. Flock-level seroprevalence against avian pneumovirus amongst Uruguayan broiler chickens. Int. J. Poult. Sci., 9: 217-220.

Gough, R.E., 2005. Poultry: Avian Pneumovirus. In: The Merck Veterinary Manual, Kahn, C.M. (Ed.). 9th Edn., Merck and Co., Whitehouse Station, pp: 2299-2300.

Hintze, J., 2008. PASS 2008 software. NCSS, Kaysville, UT.

IDEXX, 2006. Monitoring mycoplasmas in poultry farms (Animal health updates). Available from: http://wnw.idexx.com/view/xhtml/en_us/livestockpoultry/newsletter/2006/200604.jsf.

Katz, M.H., 1999. Multivariable Analysis: A Practical Guide for Clinicians. Cambridge University Press, Cambridge.

Ministerio de Ganadería Agricultura y Pesca, 2009. Division Contralor de Semovientes (DI.CO.SE.). Montevideo. Available from: http://wnw.mgap.gub. uy/dgsg/D $\mid C O S E / d i c o s e . h t m$ (in Spanish).

Noordhuizen, J.P.T.M., K. Frankena, M.V. Thrusfield and E.A.M. Graat, 2001. Application of quantitative methods in veterinary epidemiology. Wageningen Pers, Wageningen. 